\title{
Discrete Least Squares and Quadrature Formulas
}

\author{
By M. Wayne Wilson
}

\begin{abstract}
The purpose of this paper is two-fold. Firstly, we explore some of the intimate connections between discrete least squares processes and quadratures. Secondly, we present an algorithm to construct Gauss-type integration formulas, and consider briefly the method proposed by Gautschi [2].
\end{abstract}

1. Introduction. Primarily, this paper is an attempt to explore the intimate connection between numerical integration rules and discrete least squares methods. If $L$ is a linear functional on $C[-1,1]$, the set of continuous functions defined on $[-1,1]$, then (Taylor $[7, \mathrm{p} .195])$ there exists a function $\alpha \in B V[-1,1]$, the set of functions of bounded variation on $[-1,1]$, such that

$$
L(f)=\int_{-1}^{1} f(t) d \alpha(t), \quad \forall f \in C[-1,1] .
$$

We call $\alpha$ the integrator of $L$.

For $\alpha \in B V[-1,1]$, we can consider a linear functional

$$
L(f) \equiv \int_{-1}^{1} f(t) d \alpha(t)
$$

or a bilinear form

$$
(f, g) \equiv \int_{-1}^{1} f(t) g(t) d \alpha(t) .
$$

Clearly, for such an $\alpha,(f, g)=L(f g)$ and $L(f)=(1, f)$ and these we call an associated pair. Note that if $\alpha$ is a step function, with a finite number of discontinuities, then $L$, and ( , ) are finite weighted sums of function values. Similarly, finite weighted sums can be represented as Stieltjes integrals, with integrators which are step functions.

For a given linear functional $L$, we call a linear functional $L_{n}$ of the form

$$
L_{n}(f)=\sum_{i=0}^{N} A_{i}^{(n)} f\left(t_{i}\right)
$$

an $(N+1)$-point degree $n$ quadrature formula for $L$ if

$$
L_{n}(p)=L(p), \quad \forall p \in P_{n},
$$

where $P_{n}$ is the set of polynomials of degree at most $n$.

Received January 28, 1969, revised June 19, 1969.

AMS Subject Classifications. Primary 6520, 6555, 4144, 4215; Secondary 3340.

Key Words and Phrases. Discrete inner product, discrete least squares, least squares approximations, numerical integration, Gaussian integration, orthogonal polynomials, construction of inner products, construction of orthogonal polynomials. 
If $x \in B V[-1,1]$, then its $k$ th moment is

$$
\mu_{k}(x)=\int_{-1}^{1} t^{k} d x(t) .
$$

Condition (1.3) is then equivalent to

$$
\mu_{k}\left(x_{N}^{n}\right)=\mu_{k}(x), \quad k=0,1, \ldots, n,
$$

where $x_{N}^{n}$ is the integrator of (1.2).

Section 2 is dedicated to the principle that formulas should use available data, if at all possible. The first result, Construction 2.1 shows how to obtain an $(N+1)$ point degree $n$ quadrature formula, utilizing a user prescribed point set. One interpretation of this construction is as a transformation, taking a degree $n$ quadrature formula with prescribed data points (for example, a known Gauss-type rule for a particular weight function), into an $(N+1)$-point degree $n$ rule, utilizing the desired $N+1$ data points, $N \geqq n$. Theorem 2.1 gives a characterization of these rules, from which a statistical interpretation is drawn.

Construction 2.1 is then used as a basis for Construction 2.2, showing how to obtain a discrete inner product, utilizing again $N+1$ user specified points, whose first $r+1$ orthogonal polynomials, $N \geqq 2 r-1$ are precisely those of a user specified continuous distribution. This allows one to use the power of discrete Fourier series expansion, utilizing data points, and the polynomial system of one's choice. An example is given.

Section 3 applies the previous construction in the computation of Gauss-type quadrature formulas. In Section 4, we consider the method of Gautschi [2], suggest a modification, and show that in some sense, he still utilizes moment data.

2. Discrete Least Squares and Quadrature Formulas. Throughout, we will consider the linear function $L$ of (1.1), with integrator $\chi$. Our first result is a method of constructing an $(N+1)$-point degree $n$ quadrature formula for $L$, using a prescribed set of data points.

CONSTRUCTION 2.1. Let $-1 \leqq t_{0}<t_{1}<\ldots<t_{N} \leqq 1$ be $N+1$ prescribed points in $[-1,1], N \geqq n$. Let $w_{i}, i=0,1, \ldots, N$ be a set of strictly positive numbers, such that

$$
\sum_{i=0}^{N} w_{i}=\mu_{0}(x) .
$$

The inner product $[, \quad]$, gicen $b y$

$$
[f, g]=\sum_{i=0}^{N} w_{i} f\left(t_{i}\right) g\left(t_{i}\right),
$$

possesses an orthonormal sequence of polynomials $q_{0}, q_{1}, \ldots, q_{N}$. Then an $(N+1)$ point degree $n$ quadrature formula for $L$ is given $b y$.

$$
L_{n}(f)=\sum_{i=0}^{N} \lambda_{i}^{(n)} f\left(t_{i}\right)
$$

where 


$$
\lambda_{i}=w_{i}\left\{1+\sum_{j=1}^{n} q_{j}\left(t_{i}\right) M_{j}\right\}, \quad i=0,1, \ldots, N
$$

and

$$
M_{j}=L\left(q_{j}\right) .
$$

Proof. For $f \in C[-1,1]$, its $n$th Fourier segment $s_{n}(f)$ is given by

$$
s_{n}(f)=\sum_{j=0}^{n}\left[f, q_{j}\right] q_{j}, \quad n \leqq N .
$$

(If $N=n, s_{n}(f)$ is the interpolation polynomial.)

Defining $L_{n}(f)$ by $L\left(s_{n}(f)\right)$, we have

$$
\begin{aligned}
L_{n}(f) & =\sum_{j=0}^{n}\left[f, q_{j}\right] M_{j} \\
& =\sum_{i=0}^{N} f\left(t_{i}\right)\left\{w_{i} \sum_{j=0}^{n} q_{j}\left(t_{i}\right) M_{j}\right\} .
\end{aligned}
$$

But (2.1) implies $[1,1]=\mu_{0}=L(1)$ so that

$$
q_{0}(t)=\frac{1}{\sqrt{ } \mu_{0}}, \text { and } M_{0}=\sqrt{ } \mu_{0} .
$$

Thus, $L_{n}(f)$ is given by (2.3)-(2.5). For $p \in P_{n}, s_{n}(p) \equiv p$, so $L_{n}(p)=L(p), \forall p \in P_{n}$, and (2.4) is indeed a quadrature formula of degree $n$. Q.E.D.

Of course, if $N=n,(2.3)$ is the unique interpolatory quadrature formula for the data points, regardless of how the numbers $w_{i}$ were chosen. We delay discussion of calculation of $\lambda_{i}^{(n)}$ to prove the following characterization of the rule.

THEOREM 2.1. For a given set of points $t_{0}, t_{1}, \ldots, t_{N}$, and a given set of weights $w_{0}, w_{1}, \ldots, w_{N}$, then amongst all possible $(N+1)$-point degree $n$ quadrature formulas for $L$, of the form

$$
Q_{n}(f)=\sum_{i=0}^{N} A_{i} f\left(t_{i}\right), \quad n \leqq N .
$$

$L_{n}$, defined by (2.3)-(2.5), is the unique formula minimizing

$$
d(\mathbf{A})=\sum_{i=0}^{N} \frac{A_{i}^{2}}{w_{i}}
$$

Proof. We express the problem as that of minimizing the norm of an element subject to linear constraints, and apply a theorem in Davis $[1$, p. 229]. For a vector $\mathbf{x}=\left(x_{0}, x_{1}, \ldots, x_{N}\right)$ in $E^{N+1}$, define an inner product $\langle\langle\rangle$,$\rangle by$

$$
\langle\langle\mathbf{x}, \mathbf{y}\rangle\rangle=\sum_{i=0}^{N} \frac{x_{i} y_{i}}{w_{i}} .
$$

Define $\mathbf{h}_{0}, \mathbf{h}_{1}, \ldots, \mathbf{h}_{n}$ by

$$
\mathbf{h}_{i}=\left(w_{0} q_{i}\left(t_{0}\right), w_{1} q_{i}\left(t_{1}\right), \ldots, w_{N} q_{i}\left(t_{N}\right)\right) .
$$

By definition, 


$$
\left\langle\left\langle\mathbf{h}_{i}, \mathbf{h}_{j}\right\rangle\right\rangle=\left[q_{i}, q_{j}\right]=\delta_{i j}, \quad i, j=0,1, \ldots, n .
$$

Now, $L_{n}(p)=L(p), \forall p \in P_{n}$ iff $L_{n}\left(q_{j}\right)=L\left(q_{j}\right), j=0,1, \ldots, n$; that is, the vector $\mathbf{A}=\left(A_{0}, A_{1}, \ldots, A_{N}\right)$ must satisfy the constraints

$$
\left\langle\left\langle\mathbf{A}, \mathbf{h}_{j}\right\rangle\right\rangle=M_{j}, \quad j=0,1, \ldots, n .
$$

Applying the theorem in Davis, the unique element $\mathbf{A}^{*}$ of $E^{N+1}$ satisfying these constraints and minimizing $d(\mathbf{A})=\langle\langle\mathbf{A}, \mathbf{A}\rangle\rangle$ is given by

$$
\mathrm{A}^{*}=\sum_{j=0}^{n} M_{j} \mathbf{h}_{j},
$$

whose ith component,

$$
A_{i}^{*}=\sum_{j=0}^{n} w_{i} q_{j}\left(t_{i}\right) M_{j},
$$

is precisely $\lambda_{i}^{(n)}$, given by (2.4). Q.E.D.

By computation, or as a corollary to the theorem in Davis, the minimum value $d\left(\mathbf{A}^{*}\right)$ is $\sum_{i=0}^{n} M_{i}^{2}$. These quadratures contain, as a special case, the quadratures described in Wilson [10], and in the author's thesis.

This characterization has the following statistical interpretation. Let each function value $f\left(t_{i}\right)$ have an error $\varepsilon_{i}$, which, considered as an independent random variable, has variance $\sigma_{i}^{2}$. Taking $w_{i}=1 / \sigma_{i}^{2}$, Theorem 2.1 shows that the variance $\sigma^{2}$

$$
\left(\sigma^{2}=\sum_{i=0}^{N} \sigma_{i}^{2} A_{i}^{2}\right)
$$

of the total error, $E$,

$$
\left(E \equiv \sum_{i=0}^{N} A_{i} \varepsilon_{i}\right) \text { of } Q_{N}
$$

is minimized if $A_{i}$ are determined according to Construction 2.1.

Turning now to the calculation of $\lambda_{i}^{(n)}$, the values $q_{j}\left(t_{i}\right)$ are obtained by generating the set $q_{0}, q_{1}, \ldots, q_{n}$ by the three-term recurrence formula. For any inner product $(, \quad)$ the orthonormal polynomials $p_{0}, p_{1}, \ldots$, and their monic forms $\tilde{p}_{0}, \tilde{p}_{1}, \ldots$ satisfy the recurrence

$$
\begin{aligned}
\tilde{p}_{-1} & \equiv 0, \quad \tilde{p}_{0}(t) \equiv 1, \quad \beta_{0} \equiv 0 \\
\tilde{p}_{i+1}(t) & =\left(t-\alpha_{i}\right) \tilde{p}_{i}-\beta_{i} \tilde{p}_{i-:}, \quad i=0,1, \ldots \quad \text { where } \\
\alpha_{i} & =\left(t \tilde{p}_{i}, \tilde{p}_{i}\right) /\left(\tilde{p}_{i}, \tilde{p}_{i}\right), \quad \beta_{i}=\left(\tilde{p}_{i}, \tilde{p_{i}}\right) /\left(\tilde{p}_{i-1}, \tilde{p}_{i-1}\right) \quad \text { and } \\
p_{i} & =\tilde{p}_{i} /\left(\tilde{p}_{i}, \tilde{p}_{i}\right)^{1 / 2}
\end{aligned}
$$

which we collectively call (2.6)

Note that the square root operation indicated above is easily avoided in computing $\lambda_{i}^{(n)}$, since

$$
q_{j}\left(t_{i}\right) M_{j}=\tilde{q}_{j}\left(t_{i}\right) L\left(\tilde{q}_{j}\right) /\left(\tilde{q}_{j}, \tilde{q}_{j}\right)
$$


The values $M_{j}=L\left(q_{j}\right)$, or alternatively $L\left(\tilde{q}_{j}\right)$, are known in principle. They may be obtained from the moments $\mu_{k}(\alpha), k=0,1, \ldots, n$, via an (unstable) recurrence given in Gautschi [2]. If the $\tilde{q}_{j}$ can be identified with a set of classical polynomials (wishful thinking in general), analytic techniques might be employed. However, if there exists some degree $n$ quadrature formula for $L$, say $Q_{n}$, then $M_{j}$ or $L\left(\tilde{q}_{j}\right)$ are easily computed. In effect, we are then transforming the known quadrature $Q_{n}$ (for example, a Gauss-type rule, involving approximately $1 / 2^{n}$ data points) into a degree $n$ formula, with $(N+1)$ specified data points, $N \geqq n$. One advantage of this method of creating quadrature formulas is that we do not require the solution of a system of equations.

This process has application in creating quadrature formulas for data arising from experimentation, where it may be impractical, if not impossible, to obtain data to fit known rules. It has the added advantage that, via Theorem 2.1, known error characteristics of data can be incorporated.

The construction can be modified to handle the more general situation, where by degree $n$ we mean that $L_{n}(f)=L(f)$ for all function $f$ in an $(n+1)$ dimensional subspace of $C[-1,1]$, spanned by $(n+1)$ linearly independent continuous functions $\varphi_{0}, \varphi_{1}, \ldots, \varphi_{n}$. The three-term recurrence formulas, which orthonormalize $1, t, t^{2}$, $\ldots, t^{n}$ are replaced by the Gram-Schmidt orthogonalization process.

Next, we consider further ways the choice of weights $w_{i}$ affect the computation of $\lambda_{i}^{(n)}$. Let $J$ be the linear functional associated with the inner product $[$,$] . That is$

$$
J(f) \equiv[1, f], \quad \forall f \in C[-1,1] .
$$

Condition (2.1) implied that $J$ was an $(N+1)$-point degree 0 quadrature formula for $L$.

Lemma 2.1. Suppose $J$ is a degree $m$ quadrature formula for $L$. Then, for $m<n$,

$$
\lambda_{i}^{(n)}=w_{i}\left\{1+\sum_{j=m+1}^{n} q_{j}\left(t_{i}\right) M_{j}\right\}
$$

while for $m \geqq n, \lambda_{i}^{(n)}=w_{i}$.

Proof. Since $J(p)=L(p), \quad \forall p \in P_{m}$, we have $M_{j}=L\left(q_{j}\right)=J\left(q_{j}\right)=\left[1, q_{j}\right]=0$, for $j=1,2, \ldots, \min (n, m)$, by definition, assumption, and orthogonality. Q.E.D.

Thus, $\lambda_{i}^{(n)}$ can be considered as $w_{i}$, with a correction factor, which takes account only of the increase in degree. If $m \geqq n, J$ is already a degree $n$ formula with the required $N+1$ data points.

If we treat (2.2) as a bilinear form, we can relax the strict positivity requirements on the weights $w_{i}$. Everything previous will hold, with nonzero weights, provided the bilinear form [ , ] will generate a set of monic "orthogonal" polynomials $\tilde{q}_{0}, \tilde{q}_{1}$, $\ldots, \tilde{q}_{n}$ via the three-term recurrence formulas (2.6). Struble [5] shows that the recurrence breaks down only if, for some $k<n,\left[\tilde{q}_{k}, \tilde{q}_{k}\right]=0$. Thus, the existence of $\tilde{q}_{0}, \tilde{q}_{1}, \ldots, \tilde{q}_{n}$ implies the existence of "orthogonal" polynomials $q_{0}, q_{1}, \ldots, q_{n-1}$, normalized in the usual fashion. We admit the possibility of $\left[\tilde{q}_{n}, \tilde{q}_{n}\right]=0$.

Starting with [ , ] and assuming $J$ is of degree $m<n_{1}<n, n_{1}$ an integer, observe that in addition to creating $L_{n}$, we could create $L_{n_{1}}$. Taking as a new set of weights $w_{i}$ the coefficient $\lambda_{i}^{\left(n_{1}\right)}$, (which need not be strictly positive), we can create another degree $n$ formula, by a second application of the construction. Unless $N=n$, (so that $L_{n}$ is the unique interpolatory quadrature for $L$ ), the two-degree $n$ formulas 
are not in general the same. This is easily seen from Theorem 2.1 , or from experimentation.

Lemma 2.2. Let ( , ), the bilinear form associated with L, possess a sequence of monic orthogonal polynomials, $\tilde{p}_{0}, \tilde{p}_{1}, \ldots, \tilde{p}_{l}$. Let $J$, the associated linear functional of $[$,$] be of degree m$. Then [ , ] possesses a sequence of monic polynomials $\tilde{q}_{0}, \tilde{q}_{1}, \ldots, \tilde{q}_{r}$, for any $r$ satisfying $r \leqq l, 2 r-1 \leqq m$.

Further, $\tilde{q}_{j}=\tilde{p}_{j}, j=0,1, \ldots, r$, and $q_{j}=p_{j}, j=0,1, \ldots, r-1$. If, in addition $r$ satisfies $2 r \leqq m, r \leqq l$, then $q_{r}=p_{r}$, provided $\left(p_{r}, p_{r}\right) \neq 0$.

Proof. From Szegö [6, Section 2.2], or Stroud and Secrest [4, Section 1.2], or directly from the recurrence formulas, the polynomial $\tilde{p}_{k}$ is determined by the moments $\mu_{j}(\alpha), \alpha$ the integrator of $L$ or $(, \quad)$, for $j=0,1, \ldots, 2 k-1$. If $\left(\tilde{p}_{k}, \tilde{p}_{k}\right) \neq 0$, $p_{k}$ is determined by $\mu_{j}(\alpha), i=0,1, \ldots, 2 k$. Since $J$ is a quadrature formula of degree $m$, its integrator has the same first $m+1$ moments as L. Q.E.D.

In the statement of the lemma, if $l$ is finite (we allow $l$ to be finite or infinite), then, unless degeneracy occurs, $p_{l}$ is also available. If $\alpha(t)$ is monotonic increasing, we have orthogonal polynomials in the classical sense, and degeneracy cannot occur.

This leads to the following

CONSTRUCTION 2.2. Let ( , ) be a bilinear form, possessing a sequence of monic orthogonal polynomials, $\tilde{p}_{0}, \tilde{p}_{1}, \ldots, \tilde{p}_{r}$. Taking $n \geqq 2 r-1, N \geqq n$, the discrete bilinear form $\langle$,$\rangle , given by$

$$
\langle f, g\rangle=\sum_{i=0}^{N} \lambda_{i}^{(n)} f\left(t_{i}\right) g\left(t_{i}\right)
$$

where $\lambda_{i}^{(n)}$ are obtained by Construction 2.1, using positive weights $w_{i}$, and prescribed points $-1 \leqq t_{0}<t_{1}<\cdots<t_{N} \leqq 1$, possesses a set of monic polynomials $\tilde{q}_{0}, \tilde{q}_{1}$, $\ldots, \tilde{q}_{l}$, where $l \geqq r$, such that $\tilde{q}_{i}=\tilde{p}_{i}, i=0,1, \ldots, r$, and $q_{i}=p_{i}, i=0,1, \ldots, r-1$. If $n \geqq 2 r$, we have, in addition, $q_{r}=p_{r}$, provided degeneracy does not occur.

Proof. Immediate from Lemma 2.2

Thus, for the given data points, $t_{0}, t_{1}, \ldots, t_{N}$, we can create coefficients $\lambda_{i}^{(n)}$, not necessarily positive, to obtain a discrete orthogonality relation for a prescribed set of (classical) polynomials.

It is interesting to note that just writing

$$
\langle f, g\rangle=\sum_{i=0}^{N} a_{i} f\left(t_{i}\right) g\left(t_{i}\right)
$$

for $N+1$ prescribed points, and imposing the $r(r+3) / 2$ constraints

$$
\begin{array}{ll}
\left\langle p_{i}, p_{j}\right\rangle=0, & 0 \leqq i \leqq j \leqq r, \\
\left\langle p_{i}, p_{i}\right\rangle=1, & i=0,1, \ldots, r-1,
\end{array}
$$

we can find coefficients $a_{i}, \mathrm{i}=0,1, \ldots, N$, for any $N \geqq 2 r-1$, which satisfy the constraints, provided that there exists an $\alpha \in B V[-1,1]$, such that

$$
\int_{-1}^{1} p_{i}(t) p_{j}(t) d \alpha(t)
$$

satisfies the same constraints, where $p_{k}$ is a polynomial, of degree $k, k=0,1, \ldots, r$.

In addition to its use in the next section, Construction 2.2 allows one to use all 
the methods of (discrete) least squares, with both the data points, and the polynomial system, of one's choice, whether or not $\langle$,$\rangle is in fact an inner product.$

As an example of the methods outlined here, take $\alpha(t)=t$ in (1.1), so that $L$ is ordinary integration. For a point set, take equidistant points, $t_{i}=-1+i / 8, i=$ $0,1, \ldots, 8$. Constructing a degree 6,9 point quadrature formula, we obtain the weights

$$
\begin{aligned}
& \lambda_{0}=\lambda_{8}=0.078747, \quad \lambda_{1}=\lambda_{7}=0.343570, \\
& \lambda_{2}=\lambda_{6}=0.185866, \quad \lambda_{3}=\lambda_{5}=0.237791, \quad \lambda_{4}=0.308052
\end{aligned}
$$

using Construction 2.1, with $w_{i}=2 / 9, i=0,1, \ldots, 8$.

The known classical polynomials of $\alpha(t)=t$ are of course, the Legendre polynomials, $P_{i}$. The orthogonal polynomials of $\langle$,$\rangle , given by Construction 2.2,$ when suitably normalized, are

$$
\begin{aligned}
& Q_{i}=P_{i}, \quad i=0,1,2,3,4, \\
& Q_{5}=P_{5}-0.380533 P_{3}, \\
& Q_{6}=P_{6}-0.475798 P_{4}-0.298990 P_{2}, \\
& Q_{7}=P_{7}-0.224238 P_{5}-0.501186 P_{3}-0.222107 P_{1}, \\
& Q_{8}=P_{8}+0.242958 P_{6}-0.536490 P_{4}-0.559967 P_{2}-0.138817 P_{0} .
\end{aligned}
$$

The normalization was to expand each $\tilde{q}_{j}$ as a Legendre series, and then to make the coefficient of the highest degree Legendre polynomial unity. The calculations were done in double precision, on an IBM 360 model 67 , although only reported to six figures.

If we expand $f \in C[-1,1]$ as a discrete Fourier series, using $\langle, \quad\rangle$,

$$
f_{n}=\sum_{j=0}^{8} a_{j} Q_{j} \text { where } a_{j}=\frac{\left\langle f, Q_{j}\right\rangle}{\left\langle Q_{j}, Q_{j}\right\rangle},
$$

then $2 a_{0}$ is the value $L(f)$, i.e., of $\int_{-1}^{1} f(t) d t$ to degree 7 accuracy. That is if $f$ is sufficiently differentiable, then there is a constant $K$, and a $\zeta \in(-1,1)$, such that $2 a_{0}-L(f)=K f^{(8)}(\zeta)$.

That the rule is actually of degree 7 , not 6 , follows from the symmetry of the points and weights.

3. Gaussian Quadrature Formulas. The preceding analysis gives rise to another approach in creating an $r$-point Gauss quadrature formula for the integral

$$
L(f)=\int_{-1}^{1} f(t) d \alpha(t)
$$

where we now assume $\alpha(t)$ is monotonic increasing, with at least $r+1$ points of increase. Recall that the more usual integral $\int_{-1}^{1} w(t) f(t) d t$ where $w(t) \geqq 0$ on $[-1,1]$, and not identically zero, can be put into the above form. If $p_{0}, p_{1}, \ldots, p_{r}$ are the orthonormal polynomials associated with $\alpha$, then, the Christoffel numbers can be calculated from

$$
C_{j}^{(r)}=\left(\sum_{i=0}^{r-1}\left[p_{i}\left(\zeta_{j}\right)\right]^{2}\right)^{-1},
$$


where $\zeta_{1}, \zeta_{2}, \ldots, \zeta_{r}$ are the zeros of $p_{r}$. If the polynomials are readily available, this is the most direct process for finding the $r$-point Gauss rule for $\alpha$.

If the polynomials are not known, and if we have available an $(N+1)$-point degree $n$ quadrature formula for $L$, where $N \geqq n \geqq 2 r-1$, we can apply Construction 2.2 , to obtain $p_{0}, p_{1}, \ldots, p_{r-1}, \tilde{p}_{r}$. Then, root finding techniques, (3.2), and improvement techniques can be applied. Gautschi [2] and [3] give details and algorithms for such techniques. This process would, under the assumptions, probably still be the most direct approach.

Gautschi [2] shows that the usual methods for finding Gauss-type rules, starting with the first $2 r$ moments, and either solving a nonlinear system, or inverting a Gram matrix, are highly ill conditioned. He proposes a scheme that obtains approximations to $p_{0}, p_{1}, \ldots, p_{r}$, and uses the zero of the $r$ th degree approximant as described earlier. Since his scheme (we consider it in more detail in Section 4) appears relatively stable, proceeding as we do from an $(N+1)$-point degree $n$ quadrature formula, and also generating approximants to $p_{0}, p_{1}, \ldots, p_{r}$, the proposed scheme should also be stable, providing the quadrature formula involved is also stable. Although we can give no formal definition of stability of a quadrature rule, practically, the coefficients should all be of the same order of magnitude with few, if any, negative coefficients. In this connection, recall that Tchakaloff [8] (see also Wilson [9]) shows that for any $n$, there does exist an $(n+1)$-point degree $n$ quadrature formula for $L$, with positive weights. One method of finding such rules has been given in Wilson [11].

Let us now consider a different problem, that of creating, for $L$, a quadrature rule of degree $n$, in a stable fashion. This does not mean the rule obtained is necessarily stable. However, if it is reasonably stable, we can proceed to the calculation of the Gauss-type rule as outlined above.

If we are extremely fortunate, we might be able to calculate analytically

$$
\int_{-1}^{1} s_{j}(t) d \alpha(t), \quad j=0,1, \ldots, n,
$$

where $s_{0}, s_{1}, \ldots, s_{n}$ are a known set of classical orthogonal polynomials, with respect to an integrator $\beta \in B V[-1,1]$. Further, let us suppose that $s_{0}, s_{1}, \ldots, s_{n}$ satisfy a discrete orthogonality relation, over a set of $N+1$ points, $N \geqq n$. (The polynomials $s_{0}, s_{1}, \ldots, s_{n}$ always have such a relation with respect to the discrete inner product formed from any $(N+1)$-point Gauss-type rule for the integrator $\beta$, $N \geqq n$.) Then Construction 2.2 will create an $(N+1)$-point degree $n$ quadrature rule for $L$, i.e. for the integrator $\alpha$.

One important example occurs when we take $s_{j}=T_{j}$, the first kind Chebyshev polynomial, where we can examine, for $N=n$, the condition of the process. Defining

$$
\sum_{i=0}^{n \prime \prime} a_{i}=\frac{1}{2} a_{0}+a_{1}+a_{2}+\ldots+a_{n-1}+\frac{1}{2} a_{n}
$$

we have the natural orthogonality

$$
\begin{aligned}
\sum_{i=0}^{n}{ }^{\prime \prime} T_{k}\left(t_{i}\right) T_{j}\left(t_{i}\right) & =0, i=j, \\
& =n, i=j=0,=n, \\
& =n / 2, i=j \neq 0, \neq n,
\end{aligned}
$$


where $t_{i}=\cos (i \pi / n), i=0,1, \ldots, n$.

Applying Construction 2.1, we obtain the quadrature rule, of degree $n$,

$$
L_{n}(f)=\sum_{i=0}^{n} A_{i}^{(n)} f\left(t_{i}\right)
$$

where

$$
A_{i}^{(n)}=\frac{2}{n} \sum_{j=0}^{n} T_{j}\left(x_{i}\right) M_{j}^{*}, \quad i=0,1, \ldots, n,
$$

and $M_{j}^{*}=L\left(T_{j}\right)$.

This is equivalent to solving the matrix system $B \lambda=\mathbf{M}^{*}$ where

$$
\lambda=\left(\begin{array}{c}
\lambda_{0} \\
\lambda_{n}
\end{array}\right), \quad \mathbf{M}^{*}=\left(\begin{array}{c}
M_{0}^{*} \\
M_{n}^{*}
\end{array}\right),
$$

and $b_{i j}=T_{i}\left(t_{j}\right), i, j=0,1, \ldots, n$.

The matrix $B$ has a known exact inverse, immediate from the discrete orthogonality condition (3.3), namely

$$
\frac{2}{n} \quad\left(\begin{array}{c}
\frac{1}{4} T_{0}\left(t_{0}\right), \frac{1}{2} T_{1}\left(t_{0}\right), \ldots, \frac{1}{2} T_{n-1}\left(t_{0}\right), \frac{1}{4} T_{n}\left(t_{0}\right) \\
\frac{1}{2} T_{0}\left(t_{1}\right), T_{1}\left(t_{1}\right), \ldots, T_{n-1}\left(t_{1}\right), \frac{1}{2} T_{n}\left(t_{1}\right) \\
\ldots \ldots \ldots \ldots \ldots \ldots \ldots \ldots \ldots \ldots \ldots \ldots \ldots \ldots \ldots \ldots \\
\frac{1}{2} T_{0}\left(t_{n-1}\right), T_{1}\left(t_{n-1}\right), \ldots, T_{n-1}\left(t_{n-1}\right), \frac{1}{2} T_{n}\left(t_{n-1}\right) \\
\frac{1}{4} T_{0}\left(t_{n}\right), \frac{1}{2} T_{1}\left(t_{n}\right), \ldots, \frac{1}{2} T_{n-1}\left(t_{n}\right), \frac{1}{4} T_{n}\left(t_{n}\right)
\end{array}\right)
$$

Using the vector norm $\|\mathbf{x}\|=\max _{i}\left|x_{i}\right|$, and the corresponding matrix norm

$$
\|B\|=\max _{i} \sum_{j}\left|a_{i j}\right|,
$$

we see, since the entries are essentially cosines, $\|B\|=n+1$ and $\left\|B^{-1}\right\| \leqq 2$ so that the condition number of the system

$$
K(B)=\|B\| \cdot\left\|B^{-1}\right\| \leqq 2(n+1) .
$$

Knowing $M_{j}^{*}$ then leads to a reasonably conditioned process for determining a $(n+1)$-point degree $n$ quadrature rule for (3.1).

The importance of this example is now apparent. Even if we cannot calculate

$$
M_{j}^{*}=\int_{-1}^{1} T_{j}(t) d \alpha(t)
$$

analytically, accurate approximation of the values, which may readily be possible, does not alter the rule obtained substantially, since the matrix system is reasonably conditioned.

If we are actually dealing with the special case $\int_{-1}^{1} f(t) w(t) d t$, where $w(t) \geqq 0$ on $[-1,1]$, it is pertinent to remark that many methods of obtaining polynomial approximations to $w(t)$, particularly minimax methods, lead directly to approximants 
of the form $\sum_{i=0}^{k} a_{i} T_{i}(x)$ where $a_{i}$ rapidly decrease in magnitude. Since $\int_{-1}^{1} T_{i}(t) T_{j}(t) d t$ can be calculated, quite accurate estimates to $M_{j}^{*}$ may be obtained.

There is one class of integrators where $M_{k}^{*}$ are clearly analytically available, namely, suppose $\alpha$ is piecewise linear. For example, suppose

$$
\int_{-1}^{1} f(t) d \alpha(t)=\int_{-1}^{-1 / 2} f(t) d t+f(0)+\int_{3 / 4}^{1} f(t) d t .
$$

Since $T_{j}(x)$ is essentially a cosine, analytic calculation is easy.

Finally, for completeness only, if moment data is available, Gautschi [2] gives recurrence formulas for generating "moments" with respect to given classical orthogonal polynomials, although, as he indicates, such recurrences are not stable.

The scheme given here is not meant to replace classical methods, or the scheme proposed by Gautschi [2], but is an alternative which, like any scheme, may or may not be practical, depending on the data available. It is eminently practical if one has an $(N+1)$-point degree $2 r-1$ quadrature scheme handy, $N \geqq 2 r-1$, or if one is easily calculated. Several situations where the latter case occurs have been outlined above.

The proposed scheme appears to show the stability of Gautschi's scheme. Finally, the proposed scheme may be applied to more general situations than Gautschi's.

4. Gautschi's Scheme. Consider now the scheme proposed by Gautschi, which is applicable in situations where $L$ is given by

$$
L(f)=\int_{-1}^{1} f(t) w(t) d t
$$

where $w(t) \geqq 0$ on $[-1,1]$. We outline his scheme, indicate a modification, and show that in some sense, his scheme is not totally free of moment data.

Assume a sequence $I_{M}$ of $(M+1)$-point quadrature formulas, of degree $n_{M}$ respectively, with points $t_{0}^{(M)}, \ldots, t_{M}^{(M)}$ in $[-1,1]$ and whose weights are positive. Although he specifically recommends two particular sequences of rules, they are not important to the description of the method, although they have great practical importance. We do require that, as $M \rightarrow \infty$, the norm of the partition $t_{0}^{(M)}, t_{1}^{(M)}, \ldots, t_{M}^{(M)}$ must go to zero.

If we write $I_{M}$ as

$$
I_{M}(f)=\sum_{i=0}^{M} \alpha_{i}^{(M)} f\left(t_{i}^{(M)}\right)
$$

then he considers the sequence of orthogonal polynomials $q_{j}^{(M)}, j=0,1, \ldots, r$, generated by the discrete inner product $[f, g]_{M}=I_{M}(w f g)$ where $w(t)$ is the weight function.

He shows the sequence of polynomials $q_{k}^{(M)}, k=0,1, \ldots, r$ converge, as $M \rightarrow x_{\text {, }}$ to the sequence $p_{k}, k=1,2, \ldots, r$, the orthonormal polynomials associated with $n(t)$.

We will define

$$
[f, g]_{M}=\alpha_{M} \sum_{i=0}^{M} \alpha_{i}^{(M)} w\left(t_{i}^{(M)}\right) f\left(t_{i}^{(M)}\right) g\left(t_{i}^{(M)}\right)
$$

and assume $x_{M}$ is such that 


$$
[1,1]_{M}=\int_{-1}^{1} w(t) d t=\mu_{0},
$$

so that the corresponding quadrature formula is degree 0 . The choice has no effect on the monic polynomials, but will affect the normalization somewhat. For some $M$ sufficiently large, he takes $q_{r}^{(M)}(t)$ as an approximation to $p_{r}(t)$ and calculates the Christoffel numbers $C_{i}^{r, M}$ from its zeros $\underset{i}{\xi r, M}$, as in (3.2).

We now describe a simple modification. For $n=2 r-1$, let $N \geqq n$, and, as above, calculate $q_{k}^{(N)}(t), k=0,1, \ldots, n$, orthonormal with respect to $[,]_{N}$. For $M>N$, we consider $[1, f]_{M}$ as the linear functional $L(f)$ of Section 2 , and using Construction 2.2 , create the orthogonal polynomials $\tilde{q}_{0}^{(M)}, \ldots, \tilde{q}_{n}^{(M)}$. Recall that the inner product $\langle$,$\rangle will correctly normalize \tilde{q}_{j}^{(M)}, j=0,1, \ldots, r-1$.

This modification has value computationally if $M \gg N$, or if we compute a sequence $M_{1}<M_{2}<\ldots$ of such sets of polynomials. In the latter case, one may examine the convergence of the weights for use in the inner product $\langle$, $\rangle$, before obtaining the $\tilde{q}_{k}^{(M)}$ polynomials. Although a similar type of procedure is easily incorporated into Gautschi's method, (by examining the convergence of the coefficients of the three-term recurrence formulas) at roughly equivalent cost, a definite advantage occurs if the initial rules $I_{M}(f)$ have some form of point economization (as his suggested rules do). This advantage accrues because at each step, the Gautschi procedure involves inner products $\left[\tilde{q}_{i}^{(M)}, \tilde{q}_{i}^{(M)}\right]_{M}, i=0,1, \ldots, r$, of differing sets of polynomials, while the modification suggested involves only inner products $\left[1, \widetilde{q}_{i}^{(N)}\right]_{M}, i=0,1$, $\ldots, n$, of a fixed set of polynomials.

However, the variation above shows that in some sense, Gautschi's algorithm still depends on moment data, namely, on $\left[1, \tilde{q}_{k}^{N}\right]_{M}, k=0,1, \ldots, n$ which is an approximation to

$$
\int_{-1}^{1} \tilde{q}_{k}^{(N)}(t) w(t) d t
$$

(However, as $M$ increases, the approximation improves.) Since Gautschi states that his procedure appears to be stable, it is conjectured that the modification will also be stable.

Acknowledgment. The author gratefully acknowledges the constructive criticism of the referees; their suggestions have led to a much smoother presentation.

IBM Scientific Center

6900 Fannin Street

Houston, Texas 77025

1. P. J. Davis, Interpolation and Approximation, Blaisdell, Waltham, Mass., 1963. MR 28 \# 393.

2. W. GAUTSCHI, "Construction of Gauss-Christoffel quadrature formulas," Math. Comp., v. 22, 1968, pp. 251-270. MR 37 \#3755.

3. W. GAUTSCHI, "Algorithm, Gaussian quadrature formulas," Comm. ACM, v. 11, 1968, pp. 432-436.

4. A. H. Stroud \& D. Secrest, Gaussian Quadrature Formulas, Prentice-Hall, Englewood Cliffs, N.J., 1966. MR 34 \# 2185.

5. G. W. Struble, "Orthogonal polynomials: Variable-signed weight functions," Numer. Math. v. 5, 1963, pp. 88-94. MR 27 \#4342. 
6. G. Szegö, Orthogonal Polynomials, 2nd ed., Amer. Math. Soc. Colloq. Publ., vol. 23, Amer. Math. Soc., Providence, R. I., 1959. MR 21 \# 5029.

7. A. E. TAYLOR, Introduction to Functional Analysis, Wiley, New York, 1958. MR 20 \# 5411.

8. V. TCHAKALOFF, "Formules de cubature méchaniques à coefficients non négatifs," Bull. Sci. Math., (2) v. 81, 1957, pp. 123-134. MR 20 \#1145.

9. M. W. Wilson, Geometric Aspects of Quadratures with Non-Negative Weights, Ph.D. Thesis, Brown University, Providence, R. I., 1969.

10. M. W. WILSON, "Necessary and sufficient conditions for equidistant quadrature formulas," SIAM J. Numer. Anal. (To appear.)

11. M. W. Wilson, "A general algorithm for nonnegative quadrature formulas," Math. Comp., v. 23,1969 , pp. $253-258$. 